\title{
Latéralisation d'une péridurale thoracique [Lateralized thoracic epidural]
}

\author{
Raphaël Paris • Laurent Raynaud, MD • \\ Yann Portron, MD $\cdot$ Elodie Schaeffer
}

Received: 19 July 2012/ Accepted: 10 September 2012/Published online: 24 October 2012

(C) Canadian Anesthesiologists' Society 2012

Un homme de 20 ans devait subir une thoraco-vidéoscopie pour talcage pleural. Il a donné son consentement pour la publication de ce cas. Avant l'induction de l'anesthésie, un cathéter (18G, extrémité fermée, trois orifices latéraux, Smiths Medical, Brisbane, Australie) est inséré de $5 \mathrm{~cm}$

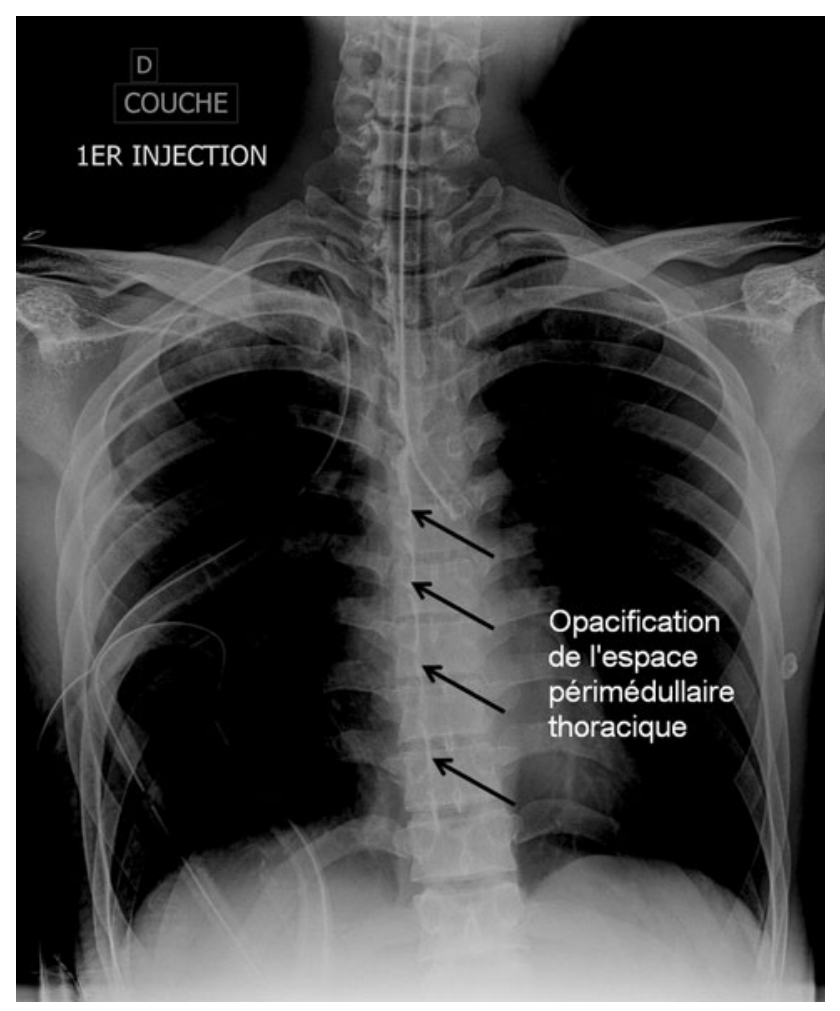

Fig. 1 Opacification latéralisée de l'espace périmédullaire thoracique

R. Paris $(\varangle)$ - L. Raynaud, MD · Y. Portron, MD · E. Schaeffer Hôpital d'Instruction des Armées Legouest, Metz, France e-mail: argaliraph@yahoo.fr dans l'espace péridural thoracique au niveau T4-T5. Après injection de $5 \mathrm{~mL}$ de lidocaïne $2 \%$ adrénalinée, une anesthésie sensitive efficace bilatérale s'étendant des métamères T3-T10 est objectivée. Après anesthésie générale et installation en décubitus latéral gauche, une perfusion continue $\left(4 \mathrm{~mL} \cdot \mathrm{h}^{-1}\right)$ de ropivacaïne $3,75 \mathrm{mg} \cdot \mathrm{mL}^{-1}$ est administrée (dose totale de $22,5 \mathrm{mg}$ ). Au réveil apparaît à droite un bloc sensitivo-moteur du membre supérieur et un syndrome de Claude Bernard-Horner (SCBH).

Les différentes hypothèses évoquées devant cette extension de l'anesthésie étaient soit une péridurale

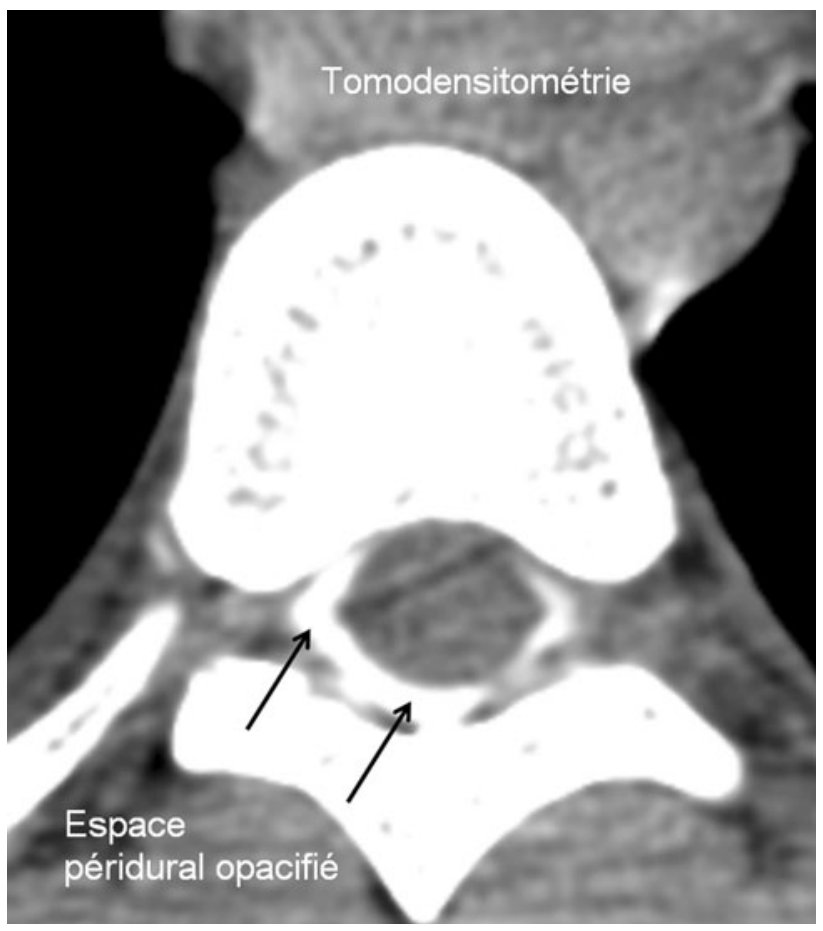

Fig. 2 Espace péridural opacifié, avec prédominance à droite 
latéralisée surdosée, soit un bloc sous-dural. Le SCBH s'explique alors par l'absence de stimulus sympathique des muscles dilatator pupillae et tarsal supérieur palpébral à cause d'une inactivation au niveau des racines nerveuses T1-T4.

L'imagerie réalisée avec opacification met en évidence une extrémité distale du cathéter au niveau de T3-T4 en postérieur, latéralisé à droite. Le cliché radiologique (Fig. 1) montre un produit de contraste (5 $\mathrm{mL}$ d'iopamidol) présent au sein de l'espace péridural et fusant le long des foramens intervertébraux de manière bilatérale prédominante à droite et le long des racines nerveuses en extra-foraminal, éliminant ainsi le diagnostic de bloc sous-dural. La tomodensitométrie (Fig. 2) montre une opacification de tout l'espace péridural, nettement plus prédominant à droite.
Devant une imagerie fortement évocatrice, un bloc sensitivo-moteur du membre supérieur et un SCBH homolatéral, le diagnostic retenu est donc celui de péridurale latéralisée. Le retrait de $2 \mathrm{~cm}$ du cathéter permet la disparition de la symptomatologie. La présence d'un bloc sous-dural aurait imposé le retrait du cathéter. Pour une nouvelle insertion, il aurait été recommandé de changer d'espace intervertébral. Le risque de bloc sous-dural réside dans la brèche arachnoïdienne transformant l'anesthésie sous-durale en une rachianesthésie.

Conflits d'intérêt Aucun. 